\title{
RUMIAS PERFECCIONISTAS EN FUNCIÓN DEL USO ESPONTÁNEO DE IMÁGENES VISUALES
}

\author{
Ma Pilar Aparicio Flores \\ Universidad de Alicante \\ pilar.aparicio@ua.es \\ María Vicent Juan \\ Universidad de Alicante \\ maria.vicent@ua.es \\ Ricardo Sanmartín López \\ Universidad de Alicante \\ ricardo.sanmartin@ua.es
}

Recepción Artículo: 27 octubre 2021 Admisión Evaluación: 27 octubre 2021 Informe Evaluador 1: 28 octubre 2021 Informe Evaluador 2: 29 octubre 2021 Aprobación Publicación: 30 octubre 2021

\section{RESUMEN}

Los Pensamientos Automáticos Perfeccionistas (PAP) son rumias basadas en la necesidad de ser perfecto, por lo que lleva consigo un alto nivel de angustia. Es por ello necesario diseñar estrategias de prevención y/o descenso de PAP. El presente trabajo partía de dos objetivos. El primero de ellos fue analizar si existen diferencias estadísticamente significativas en los factores del Uso Espontáneo de Imágenes Mentales (UEIP) en función de las altas o bajas puntuaciones de los distintos factores de PAP. El segundo de ellos analizar la probabilidad de presentar PAP en función de los factores del UEIP. Para ello se obtuvo una muestra de 791 participantes $(M=$ 22.5; $D E=5.21$ ) y se utilizaron como instrumentos de medida la Perfectionism Cognitions Inventory (PCI) y la Spontaneous Use of Imagery Scale (SUIS). Los hallazgos arrojaron diferencias estadísticamente significativas en Demandas y Esfuerzos Perfeccionistas, así como en el total de la PCI en todos los factores del UEIP, así como el total de la SUIS. Asimismo, Ios resultados indicaron que conforme aumenta la puntuación en las distintas dimensiones de la SUIS, aumenta la probabilidad tanto de presentar DP, como EP y PAP en general. En conclusión, el presente estudio muestra peculiaridades específicas de los PAP que permiten diseñar estrategias de prevención sobre estas rumias perfeccionistas.

Palabras clave: pensamientos automáticos perfeccionistas; uso espontáneo de imágenes mentales, universitarios; intervención

\section{ABSTRACT}

Perfectionist automatic thoughts and spontaneous use of visual images in Spanish undergraduates. Perfectionist Automatic Thoughts (PAT) are rumors based on the need to be perfect, so it carries with 


\section{RUMIAS PERFECCIONISTAS EN FUNCIÓN DEL USO ESPONTÁNEO DE IMÁGENES VISUALES}

it a high level of distress. It is therefore necessary to design strategies for the prevention and reduction of PAT. The present work was based on two objectives. The first of them was to analyze whether there are statistically significant differences in the Spontaneous Use of Mental Images (SUMI) factors based on the high or low scores of the different PAT factors. The second of them analyze the probability of presenting PAT as a function of the SUMI factors. For this, a sample of 791 participants $(M=22.5 ; S D=5.21)$ was obtained and the Perfectionism Cognitions Inventory (PCI) and the Spontaneous Use of Imagery Scale (SUIS) were used as measurement instruments. The findings yielded statistically significant differences in Perfectionist Demands and Perfectionist Strivings, as well as in the total of the PCI in all the factors of the SUMI, as well as the total of the SUIS. Likewise, the results indicated that as the score increases in the different dimensions of the SUIS, the probability of presenting PD, PS and PAT in general increases. In conclusion, this study shows specific peculiarities of PAT that allow the design of prevention strategies on these perfectionist rumors.

Keywords: perfectionist automatic thoughts; spontaneous use of mental images; undergraduates; intervention

\section{INTRODUCCIÓN}

El esfuerzo incesante para alcanzar la perfección es cada vez más frecuente en la población debido a las altas demandas marcadas por la sociedad (Vicent et al., 2021). Según la literatura previa, el perfeccionismo puede ser adaptativo 0 desadaptativo. Sin embargo, la mayoría de estudios se decantan por la parte negativa del rasgo debido a su vínculo con el estrés, ansiedad social (Mohammadi y Roshan-Chasli, 2020), ansiedad escolar (ÁlvarezTeruel et al., 2021), agresividad (Ruiz-Esteban et al., 2021) o rumia autocrítica (García-Fernández, et. al 2015; Fearn et al., 2021) entre otras variables. De hecho, existen unas rumias constantes basadas en la divergencia entre el "yo real" y el "yo ideal" (Hewitt y Genest, 1990) conocidas como Pensamientos Automáticos Perfeccionistas (PAP), las cuales se envuelven en un estado de pesimismo y afecto negativo (Flett et al., 2012) debido a la elevada angustia (Lyubomirsky et al., 2015) que desencadenan estas reflexiones reiterativas.

Pese a contar con numerosos estudios sobre el perfeccionismo, Ios PAP presentan una menor investigación. De hecho, todavía se carece de estudios especíicicos que muestren cómo trabajar específicamente este tipo de rumias. Teniendo en cuenta estudios como el de Wigert et al. (2012), el cual muestra que el perfeccionismo adaptativo se vincula a la creatividad, sería conveniente observar cómo intervienen estas rumias en variables de carácter visual, con el fin de poder diseñar estrategias de prevención y/o disminución de estas rumias.

En este sentido se encuentra el uso espontáneo de imágenes mentales (UEIM), el cual se entiende como la capacidad espontánea de imaginar frecuentemente una imagen mental cuando se está pensando en un elemento 0 situación cotidiana (Nelis et al., 2014). El UEIM es un amplificador emocional debido a que funciona como una predicción futura 0 de recuerdos en la mente del lector (Andrade et al., 2013), que puede desencadenar tanto aspectos positivos, basados en la creatividad (Daniels-McGhee y Davis, 1994) entre otras variables, como negativos tales como la ansiedad social (Vassilopoulos y Moberly, 2013). Asimismo, se ha observado que en personas con elevados niveles de perfeccionismo el UEIM genera un alto grado de angustia si esas imágenes son rumiantes y de carácter negativo (Lee et al., 2011). Sin embargo, y pese a haber un estudio específico que afirma el vínculo entre el UEIM y los PAP en universitarios (Aparicio-Flores et al., 2020), sería conveniente observar cómo afecta cada uno de los factores UEIM en cada dimensión de PAP, así como analizar la probabilidad de presentar un mayor UEIM sobre el aumento de PAP.

\section{OBJETIVOS DE LA INVESTIGACIÓN}

El objetivo del trabajo fue observar la asociación entre el UEIM y los PAP en universitarios españoles. De manera más precisa, los objetivos específicos del trabajo se concretan en: a) analizar si existen diferencias estadísticamente significativas en las dimensiones del UEIM y los factores de PAP; y b) evaluar la probabilidad de aumento del UEIM sobre el aumento de los PAP. 


\section{MUESTRA Y/O PARTICIPANTES}

La muestra se compuso por 791 participantes, matriculados en $4^{\circ}$ curso del Grado universitario en maestro de Educación Infantil o maestro de Educación Primaria de la Universidad de Alicante. La muestra tenía edades comprendidas entre 19 y $24(M=22.5 ; D E=5.21)$. Del mismo modo, un total del $68 \%$ eran mujeres.

\section{METODOLOGÍA Y/O INSTRUMENTOS UTILIZADOS}

\section{Instrumentos}

Perfectionism Cognitions Inventory (PCI): El PCI validado en España (Esteve-Faubel et al., 2020) se trata de una escala multidimensional compuesta por 17 ítems con 5 opciones de respuesta ( 1 = De ningún modo; $5=$ Todo el tiempo) y tres dimensiones: F1. Preocupaciones Perfeccionistas (PP), con 6 ítems, que evalúan las rumias de intranquilidad en la ejecución de una tarea y la angustia de no obtener el rendimiento deseado (ej.: Debería ser perfecto); F2. Demandas Perfeccionistas (DP), con 4 ítems, que miden reflexiones de demanda y automejora (ej.: Necesito hacerlo mejor); F3. Esfuerzos Perfeccionistas (EP), con 7 ítems, que valoran la crítica excesiva sobre el esfuerzo realizado para alcanzar la perfección (ej.: Tengo que trabajar duro todo el tiempo).

La fiabilidad de la escala fue adecuada tanto para el total del PCI ( =.83) como para sus tres dimensiones ( $=.71, .86, .88$, respectivamente).

Spontaneous Use of Imagery Scale (SUIS; Kosslyn et al., 1998). La SUIS validada en España (AparicioFlores et al., 2018) se trata una escala tipo Likert de 9 ítems y 5 opciones de respuesta ( $1=N a d a$ apropiado, $5=$ Completamente apropiado). Esta escala mide el UEIM desde tres factores que evalúa el uso espontáneo de imágenes mentales desde 3 dimensiones: F1: Capacidad Visual-Espacial (CVE), con 3 ítems, evalúa la inteligencia visual-espacial (e.g.: Cuando pienso en visitar a alguien de mi familia, casi siempre tengo una clara imagen mental de él o ella); F2: Construcción de Imágenes Mentales por Asociación (CIMA), con 3 ítems, evalúa supuestas imágenes creadas por asociación de imágenes previas (e.g.: Si viera un accidente de coche, visualizaría lo que había pasado cuando más tarde intentase recordar los detalles); y F3: Uso de Imágenes Predictivas por Experiencia (UIPE), con 3 ítems, mide la elaboración de futuras imágenes debido a experiencias vividas anteriormente (e.g.: Cuando voy a vestirme me imagino primero cómo voy a estar usando diferentes combinaciones de ropa).

La fiabilidad de la fue adecuada tanto para el total de la escala $(=.75)$ como para sus tres dimensiones ( $=.71$, $.72, .73$, respectivamente).

\section{Procedimiento}

Primeramente, el equipo investigador se entrevistó con el profesorado de los estudiantes participantes, con la finalidad de informarles del estudio y solicitar su colaboración. Posteriormente, tras solicitar su consentimiento informado, se administraron las escalas, aclarando que se trataba de un proceso voluntario y anónimo. El proceso duró alrededor de 30 minutos y se contó con la presencia de un investigador y el profesorado encargado.

\section{Análisis de datos}

La evaluación de la significatividad del UEIM y los PAP se realizó mediante el índice $d$, y se analizó bajo los criterios propuestos por Cohen (1988), el cual estima un tamaño del efecto pequeño (.20 a .49), moderado (.50 a .79), y grande $(<.80)$.

El análisis de probabilidad predictiva se realizó mediante regresión logística (pasos hacia adelante) basado en el estadístico de Wald, estimada por el estadístico odd ratio (OR).

La $R^{2}$ de Nagelkerke, informa del \% de varianza explicada por el modelo (Nagelkerke, 1991), y el de casos clasificados correctamente. Por otro lado, las variables PP, DP y EP fueron dicotomizadas en bajas (centil <25) y altas puntuaciones (centil >75). 
RUMIAS PERFECCIONISTAS EN FUNCIÓN DEL USO ESPONTÁNEO DE IMÁGENES VISUALES

\section{RESULTADOS ALCANZADOS}

\section{Diferencias en UEIM con altas y bajas puntuaciones en PP}

Los resultados observados desde la Tabla 1 y Figura 1 no arrojan diferencias estadísticamente significativas para ninguna de las dimensiones de la SUIS y las PP.

Tabla 1.Diferencias en UEIM con altas y bajas puntuaciones en PP

\begin{tabular}{lcccccccccc}
\hline \multicolumn{1}{c}{$P$ P } & $\begin{array}{c}\text { Prueba de } \\
\text { Levene }\end{array}$ & \multicolumn{2}{c}{$\begin{array}{c}\text { Bajas } \\
\text { puntuaciones }\end{array}$} & $\begin{array}{c}\text { Altas } \\
\text { puntuaciones }\end{array}$ & \multicolumn{3}{c}{$\begin{array}{c}\text { Significación } \\
\text { Estadística }\end{array}$} \\
\hline Dimensiones & $F$ & $p$ & $M$ & $D T$ & $M$ & $D T$ & $t$ & $g . l$ & $p$ & $d$ \\
\hline CVE & 2.61 & .107 & 11.12 & 2.08 & 11.05 & 2.41 & .28 & 388 & .774 & - \\
\hline CIMA & .24 & .624 & 9.71 & 2.48 & 10.06 & 2.49 & -1.37 & 388 & .169 & - \\
\hline UIPE & 1.18 & .277 & 11.67 & 2.51 & 11.50 & 2.63 & .66 & 388 & .509 & - \\
\hline SUIStotal & 3.62 & .058 & 32.50 & 5.22 & 32.61 & 5.80 & -.19 & 388 & .847 & -
\end{tabular}

Nota $:$ CVE= Capacidad Visual-Espacial; CIMA= Construcción de Imágenes Mentales por Asociación; $\mathrm{UIPE}=$ Uso de Imágenes Predictivas por Experiencia.

Figura 1. Diferencias en UEIM en universitarios con altas $y$ bajas puntuaciones en $P P .{ }^{\star} p<.05,{ }^{* \star} p<.01,{ }^{* \star \star} p<.001$

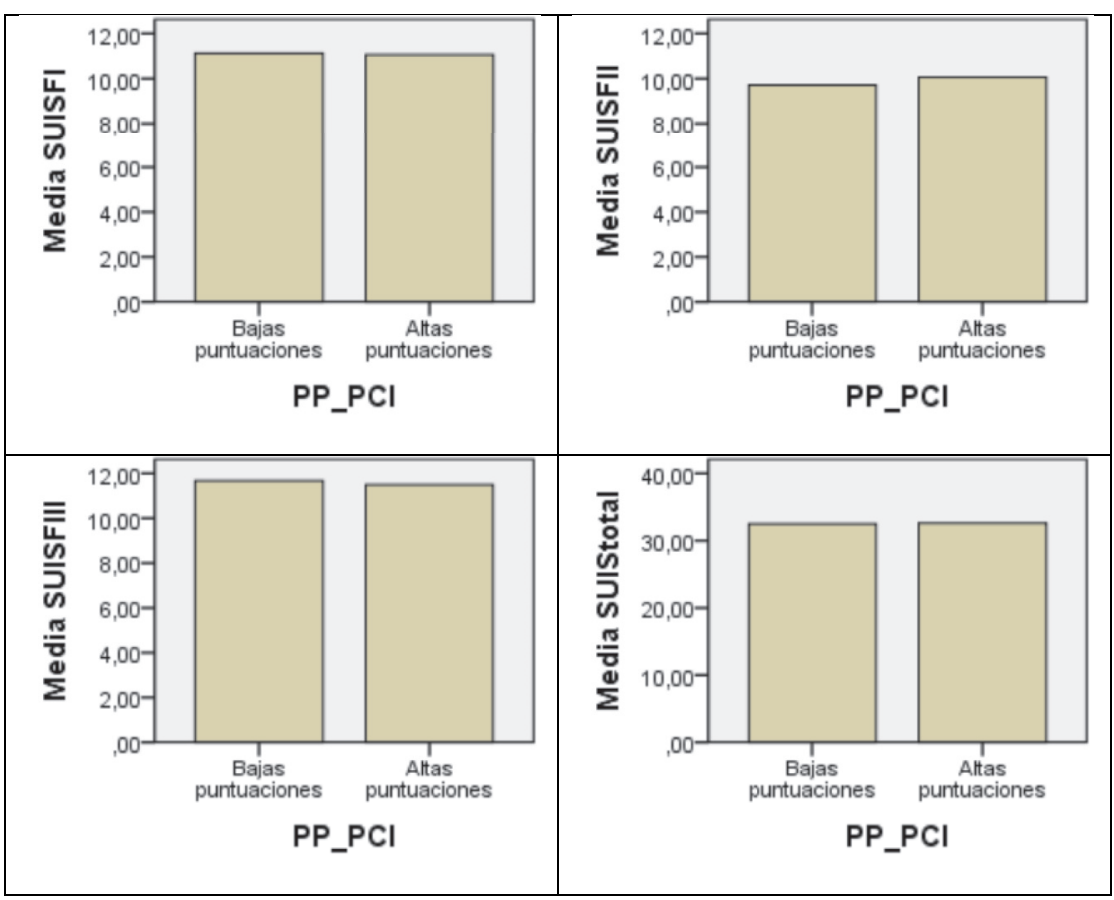




\section{Diferencias en UEIM con altas y bajas puntuaciones en DP}

Los resultados observados en la Tabla 2 y Figura 2 muestran diferencias significativas de baja y moderada magnitud para todos los factores ( $d=$ entre .36 y .48) y el total de la SUIS $(d=.57)$ en DP. Concretamente, se observan mayores puntuaciones de CVE, CIMA, UIPE y el total de la escala en altas DP frente a universitarios con bajas DP.

Tabla 2.Diferencias en UEIM con altas y bajas puntuaciones en DP

\begin{tabular}{lcccccccccc}
\hline DP & $\begin{array}{c}\text { Prueba de } \\
\text { Levene }\end{array}$ & $\begin{array}{c}\text { Bajas } \\
\text { puntuaciones }\end{array}$ & $\begin{array}{c}\text { Altas } \\
\text { puntuaciones }\end{array}$ & \multicolumn{3}{c}{$\begin{array}{c}\text { Significación } \\
\text { Estadística }\end{array}$} \\
\hline Dimensiones & $F$ & $p$ & $M$ & $D T$ & $M$ & $D T$ & $t$ & $g . l$ & $p$ & $d$ \\
\hline CVE & .84 & .359 & 10.29 & 2.23 & 11.25 & 2.09 & -4.40 & 416 & $<.001$ & .45 \\
\hline CIMA & 5.71 & .017 & 9.35 & 2.39 & 10.27 & 2.67 & -3.61 & 354.50 & $<.001$ & .36 \\
\hline UIPE & 1.76 & .185 & 10.33 & 2.67 & 11.57 & 2.55 & -4.71 & 416 & $<.001$ & .48 \\
\hline SUIStotal & .28 & .596 & 29.98 & 5.38 & 33.09 & 5.50 & -5.63 & 416 & $<.001$ & .57
\end{tabular}

Nota CVE= Capacidad Visual-Espacial; CIMA= Construcción de Imágenes Mentales por Asociación; $\mathrm{UIPE}=$ Uso de Imágenes Predictivas por Experiencia.

Figura 2. Diferencias en UEIM en universitarios con altas y bajas puntuaciones en $D P{ }^{*}{ }^{*} p<.05,{ }^{\star \star} p<.01,{ }^{* \star *} p<.001$

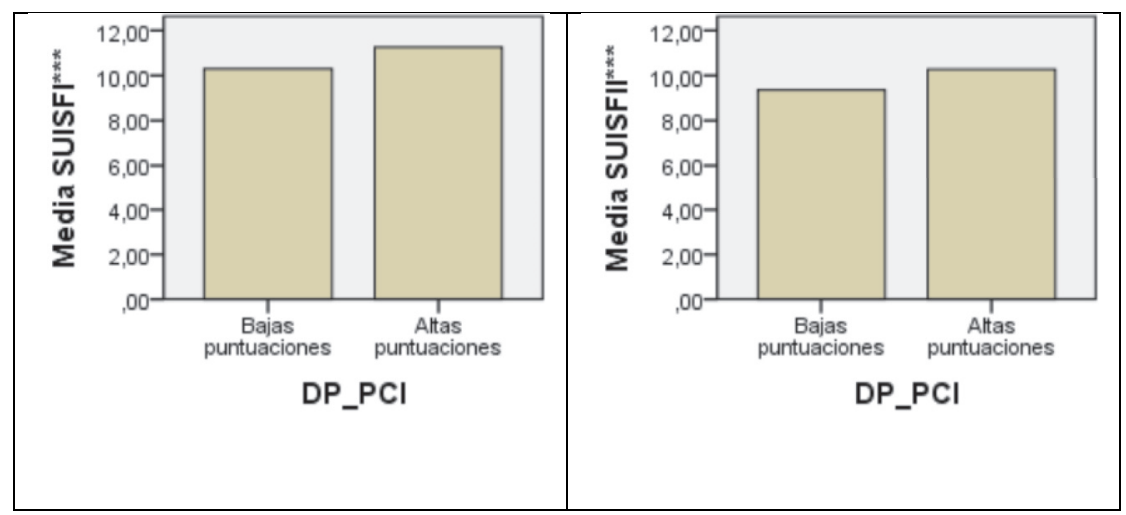




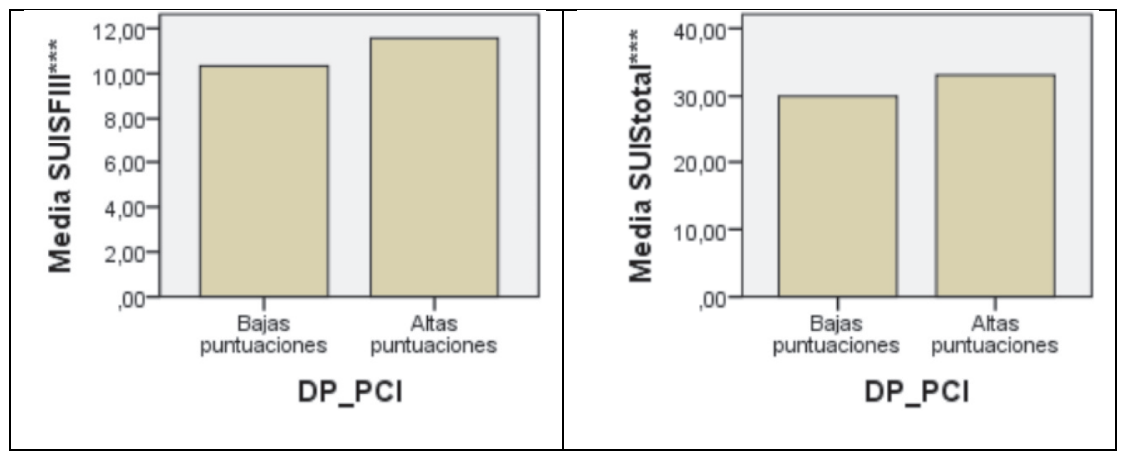

\section{Regresión logística binaria para la probabilidad de presentar alta puntuación en DP en función del UEIM}

Los resultados hallados permiten hacer una estimación correcta del $65.1 \%$ de los casos $\left(\chi^{2}=18.78 ; p=<.05\right)$ para la CVE, del $62.2 \%$ de los casos $\left(\chi^{2}=12.16 ; p=<.05\right)$ para la CIMA, del 65.6\% para el UIPE $\left(\chi^{2}=21.16 ; p=\right.$ $<.05)$, y para el total de la SUIS $\left(\chi^{2}=65.6 ; p=<.05\right)$. El valor de ajuste de los modelos ( $\mathrm{R}^{2}$ Nagelkerke) está entre .04 para la CIMA y .10 para el total de la escala (véase Tabla 3).

Los valores de la odd ratio $(O R)$ fueron superiores a 1 , Io que indica que a medida que aumenta la puntuación en CVE, CIMA, UIPE y el total de la SUIS aumenta la probabilidad de presentar altas puntuaciones en DP, con valores entre 1.10 para el total de la SUIS y 1.22 para la CVE.

Tabla 3.Regresión logística binaria para la probabilidad de presentar altas DP en función de las dimensiones de la SUIS y el total de la escala.

\begin{tabular}{|c|c|c|c|c|c|c|c|c|c|}
\hline Variable & & $\chi^{2}$ & $R^{2}$ & $B$ & E.T. & Wald & $p$ & $O R$ & I.C. $95 \%$ \\
\hline \multirow[t]{2}{*}{ CVE } & $\begin{array}{l}\text { Clasificados } \\
\text { correc.: } 65.1 \%\end{array}$ & 18.78 & .06 & .20 & .04 & 17.76 & .000 & 1,22 & $1.12-1.35$ \\
\hline & Constante & & & -1.68 & .52 & 10.13 & .001 & .18 & \\
\hline \multirow[t]{2}{*}{ CIMA } & $\begin{array}{l}\text { Clasificados } \\
\text { correc.: } 62.2 \%\end{array}$ & 12.16 & .04 & .13 & .04 & 11.71 & .001 & 1,14 & $1.06-1.24$ \\
\hline & Constante & & & -.83 & .40 & 4.24 & .039 & .43 & \\
\hline \multirow[t]{2}{*}{ UIPE } & $\begin{array}{l}\text { Clasificados } \\
\text { correc.: } 65.6 \%\end{array}$ & 21.16 & .07 & .17 & .04 & 19.95 & .000 & 1,19 & $1.10-1.29$ \\
\hline & Constante & & & -1.42 & .44 & 10.30 & .001 & .24 & \\
\hline \multirow[t]{2}{*}{ SUIStotal } & $\begin{array}{l}\text { Clasificados } \\
\text { correc.: } 65.6 \%\end{array}$ & 20.05 & .10 & .10 & .019 & 27.38 & .000 & 1,10 & $1.06-1.15$ \\
\hline & & & & -2.69 & .618 & 18.94 & .000 & .06 & \\
\hline
\end{tabular}

\footnotetext{
Nota: $\chi^{2}=$ Chi cuadrado; $R^{2}=$ Cuadrado de Nagelkerke; $B=$ Coeficiente de regresión; E.T.= Error estándar; Wald $=$ Prueba de Wald; $p=$ Probabilidad; $O R=$ Odd ratio; $I . C .=$ Intervalo de confianza al $95 \%$; $\mathrm{CVE}=$ Capacidad Visual-Espacial; CIMA= Construcción de Imágenes Mentales por Asociación; UIPE= Uso de Imágenes Predictivas por Experiencia.
}

\section{Diferencias en UEIM con altas y bajas puntuaciones en EP}

Los hallazgos que muestran la Tabla 4 y Figura 3 arrojan diferencias significativas de pequeña magnitud para todas las dimensiones ( $d=$ entre.19 y .21) y el total de la SUIS ( $d=.26)$, indicando que los universitarios con altas 
puntuaciones en EP puntúan significativamente más alto en todas las dimensiones de la SUIS que sus iguales con bajas puntuaciones en EP.

Tabla 4.Diferencias en UEIM con altas y bajas puntuaciones en EP

\begin{tabular}{lcccccccccc}
\hline \multicolumn{1}{c}{ EP } & $\begin{array}{c}\text { Prueba de } \\
\text { Levene }\end{array}$ & \multicolumn{2}{c}{$\begin{array}{c}\text { Bajas } \\
\text { puntuaciones }\end{array}$} & \multicolumn{2}{c}{$\begin{array}{c}\text { Altas } \\
\text { puntuaciones }\end{array}$} & \multicolumn{4}{c}{$\begin{array}{c}\text { Significación } \\
\text { Estadística }\end{array}$} \\
\hline Dimensiones & $F$ & $p$ & $M$ & $D T$ & $M$ & $D T$ & $t$ & $g . l$ & $p$ & $d$ \\
\hline CVE & .06 & .807 & 10.82 & 2.38 & 11.26 & 2.32 & -1.99 & 472 & .047 & .19 \\
\hline CIMA & .19 & .655 & 9.63 & 2.37 & 10.15 & 2.46 & -2.27 & 472 & .024 & .21 \\
\hline UIPE & 1.24 & .265 & 11.20 & 2.85 & 11.70 & 2.52 & -1.99 & 472 & .047 & .19 \\
\hline SUIStotal & .18 & .668 & 31.65 & 5.83 & 33.12 & 5.45 & -2.76 & 472 & .006 & .26 \\
\hline
\end{tabular}

Nota $:$ CVE = Capacidad Visual-Espacial; CIMA= Construcción de Imágenes Mentales por Asociación; $\mathrm{UIPE}=$ Uso de Imágenes Predictivas por Experiencia.

Figura 3. Diferencias en UEIM en universitarios con altas y bajas puntuaciones en $E P .{ }^{*} p<.05,{ }^{* *} p<.01,{ }^{* * *} p<.001$

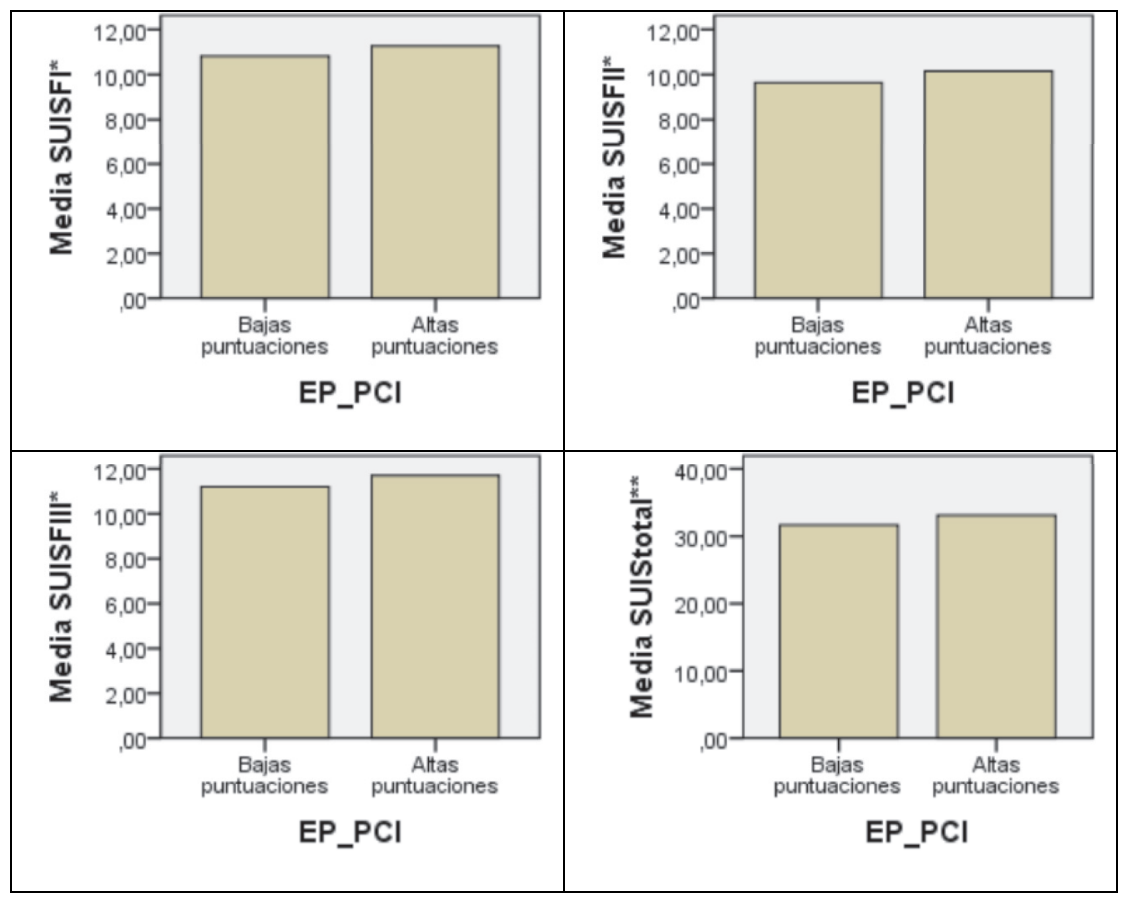




\section{RUMIAS PERFECCIONISTAS EN FUNCIÓN DEL USO ESPONTÁNEO DE IMÁGENES VISUALES}

\section{Regresión logística binaria para la probabilidad de presentar alta puntuación EP en función del UEIM}

Los hallazgos observados en la Tabla 5 permiten hacer una estimación correcta del $62 \%$ de los casos $\left(\chi^{2}=\right.$ 3.94; $p=<.05)$ para la CVE, del $61.2 \%$ de los casos $\left(\chi^{2}=5.15 ; p=<.05\right)$ para la CIMA, del $61.6 \%$ de los casos $\left(\chi^{2}=3.93 ; p=<.05\right)$ para el UIPE, y del $64.1 \%$ de los casos $\left(\chi^{2}=7.54 ; p=<.05\right)$ para el total de la SUIS.

El valor de ajuste de los modelos ( $R^{2}$ Nagelkerke) comprende entre .01 para la CVE y UIPE y 02 para la CIMA y el total de la SUIS.

Los valores de la odd ratio $(O R)$ fue superior a 1 , indicando que a medida que aumenta la puntuación en todas las dimensiones y el total de la SUIS aumenta la probabilidad de presentar altas puntuaciones en EP, con valores entre 1.04 para el total de la escala y 1.09 para la CIMA.

Tabla 5.Regresión logística binaria para la probabilidad de presentar alto EP en función de las dimensiones de la SUIS y el total de la escala.

\begin{tabular}{|c|c|c|c|c|c|c|c|c|c|}
\hline Variable & & $\chi^{2}$ & $R^{2}$ & $B$ & E.T. & Wald & $p$ & $O R$ & I.C. $95 \%$ \\
\hline \multirow[t]{2}{*}{ CVE } & $\begin{array}{l}\text { Clasificados } \\
\text { correc.: } 62 \%\end{array}$ & 3.94 & .01 & .08 & .04 & 3.92 & .048 & 1,08 & $1.01-1.17$ \\
\hline & Constante & & & -.39 & .45 & .74 & .389 & .67 & \\
\hline \multirow[t]{2}{*}{ CIMA } & $\begin{array}{l}\text { Clasificados } \\
\text { correc.: } 61.2 \%\end{array}$ & 5.15 & .02 & .08 & .03 & 5.07 & .024 & 1,09 & $1.01-1.18$ \\
\hline & Constante & & & -.38 & .39 & .93 & .333 & .68 & \\
\hline \multirow[t]{2}{*}{ UIPE } & $\begin{array}{l}\text { Clasificados } \\
\text { correc.: } 61.6 \%\end{array}$ & 3.93 & .01 & .07 & .03 & 3.91 & .048 & 1,07 & $1.01-1.15$ \\
\hline & Constante & & & -.31 & .41 & .56 & .451 & .73 & \\
\hline \multirow[t]{2}{*}{ SUIStotal } & $\begin{array}{l}\text { Clasificados } \\
\text { correc.: } 64.1 \%\end{array}$ & 7.54 & .02 & .04 & .01 & 7.42 & .006 & 1,04 & $1.01-1.08$ \\
\hline & & & & -1.01 & .55 & 3.28 & .070 & .36 & \\
\hline
\end{tabular}

Nota: $\chi^{2}=$ Chi cuadrado; $R^{2}=$ Cuadrado de Nagelkerke; $B=$ Coeficiente de regresión; E.T.= Error estándar; Wald $=$ Prueba de Wald; $p=$ Probabilidad; $O R=$ Odd ratio; I.C. = Intervalo de confianza al 95\%; CVE= Capacidad Visual-Espacial; CIMA= Construcción de Imágenes Mentales por Asociación; UIPE= Uso de Imágenes Predictivas por Experiencia.

\section{Diferencias en UEIM con altas y bajas puntuaciones en el total del PCI}

La Tabla 6 y Figura 4 arrojan diferencias significativas de pequeña magnitud para todas las dimensiones ( $d=$ entre 22 y .29) y el total de la SUIS ( $d=.34$ ), indicando que los participantes con altas puntuaciones en PAP puntúan significativamente más alto en CVE, CIMA, UIPE y el total del UEIM que los universitarios con bajas puntuaciones en PAP. 
Tabla 6.Diferencias en UEIM con altas y bajas puntuaciones en el total del PCI

\begin{tabular}{lcccccccccc}
\hline total & $\begin{array}{c}\text { Prueba de } \\
\text { Levene }\end{array}$ & \multicolumn{2}{c}{$\begin{array}{c}\text { Bajas } \\
\text { puntuaciones }\end{array}$} & \multicolumn{2}{c}{$\begin{array}{c}\text { Altas } \\
\text { puntuaciones }\end{array}$} & \multicolumn{4}{c}{$\begin{array}{c}\text { Significación } \\
\text { Estadística }\end{array}$} \\
\hline Dimensiones & $F$ & $p$ & $M$ & $D T$ & $M$ & $D T$ & $t$ & $g . l$ & $p$ & $d$ \\
\hline CVE & .01 & .979 & 10.59 & 2.36 & 11.26 & 2.36 & -2.80 & 392 & .005 & .28 \\
\hline CIMA & .01 & .942 & 9.46 & 2.59 & 10.22 & 2.61 & -2.90 & 392 & .004 & .29 \\
\hline UIPE & 1.88 & .170 & 11.07 & 2.92 & 11.69 & 2.64 & -2.21 & 392 & .028 & .22 \\
\hline SUIStotal & .28 & .594 & 31.13 & 6.14 & 33.18 & 5.79 & -3.41 & 392 & .001 & .34
\end{tabular}

Nota $:$ CVE = Capacidad Visual-Espacial; CIMA= Construcción de Imágenes Mentales por Asociación; $\mathrm{UIPE}=$ Uso de Imágenes Predictivas por Experiencia.

Figura 4. Diferencias en UEIM en universitarios con altas y bajas puntuaciones en el total del PCl. ${ }^{*} p<.05,{ }^{* *} p<.01,{ }^{* * *} p<.001$

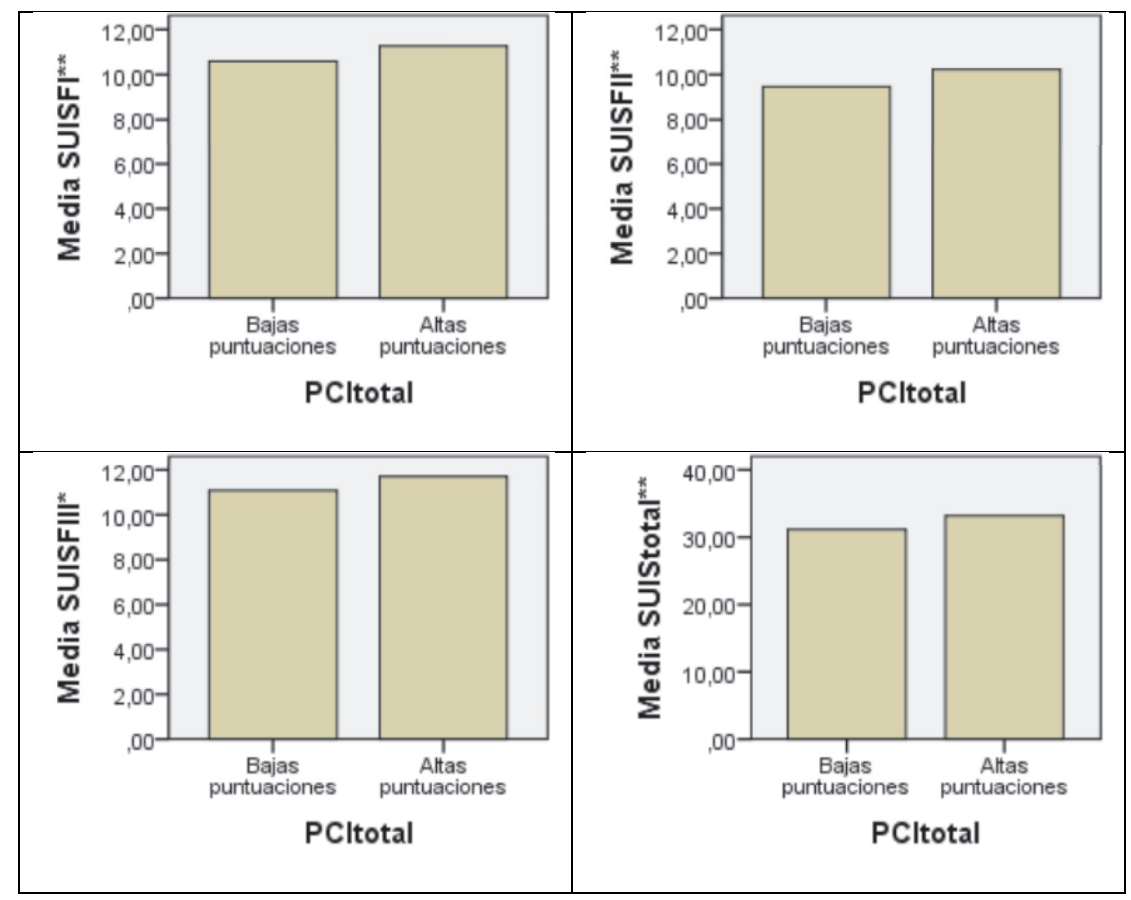

\section{Regresión logística binaria para la probabilidad de presentar alta puntuación en el total del PCI en función del UEIM}

Los resultados de la Tabla 7 permiten hacer una estimación correcta del $55.3 \%$ de los casos $\left(\chi^{2}=7.483 ; p=\right.$ $<.05)$ para la CVE, del $57.4 \%$ de los casos $\left(\chi^{2}=8.39 ; p=<.05\right)$ para la CIMA, del $58.4 \%$ de los casos $\left(\chi^{2}=4.86\right.$; $p=<.05)$ para el UIPE y del $58.4 \%$ de los casos $\left(\chi^{2}=11.52 ; p=<.05\right)$ para el total de la SUIS. 


\section{RUMIAS PERFECCIONISTAS EN FUNCIÓN DEL USO ESPONTÁNEO DE IMÁGENES VISUALES}

SUIS.

El valor de ajuste de los modelos ( $\mathrm{R}^{2}$ Nagelkerke) comprende entre .02 para el UIPE y .04 para el total de la

Los valores de la odd ratio (OR) fuer superior a 1, indicando que a medida que aumenta la puntuación en todos los factores y el total de la SUIS aumenta la probabilidad de presentar elevadas puntuaciones en el total de la PCI, con valores entre 1.04 para el UIPE y 1.12 para la CVE y CIMA.

Tabla 7.Regresión logística binaria para la probabilidad de presentar alto PCltotal en función de las dimensiones de la SUIS y el total de la escala.

\begin{tabular}{|c|c|c|c|c|c|c|c|c|c|}
\hline Variable & & $\chi^{2}$ & $R^{2}$ & $B$ & E.T. & Wald & $p$ & $O R$ & I.C. $95 \%$ \\
\hline \multirow[t]{2}{*}{ CVE } & $\begin{array}{l}\text { Clasificados } \\
\text { correc.: } 55.3 \%\end{array}$ & 7.83 & .03 & .12 & .04 & 7.59 & .006 & 1,12 & $1.04-1.23$ \\
\hline & Constante & & & -1.16 & .48 & 5.68 & .017 & .31 & \\
\hline \multirow[t]{2}{*}{ CIMA } & $\begin{array}{l}\text { Clasificados } \\
\text { correc.: } 57.4 \%\end{array}$ & 8.39 & .03 & .11 & .04 & 8.15 & .004 & 1,12 & $1.04-1.21$ \\
\hline & Constante & & & -.96 & .40 & 5.71 & .017 & .38 & \\
\hline \multirow[t]{2}{*}{ UIPE } & $\begin{array}{l}\text { Clasificados } \\
\text { correc.: } 58.4 \%\end{array}$ & 4.86 & .02 & .08 & .03 & 4.77 & .029 & 1,04 & $1.01-1.17$ \\
\hline & Constante & & & -.76 & .43 & 3.13 & .077 & .46 & \\
\hline \multirow[t]{2}{*}{ SUIStotal } & $\begin{array}{l}\text { Clasificados } \\
\text { correc.: } 58.4 \%\end{array}$ & 11.52 & .04 & .05 & .01 & 11.01 & .001 & 1,06 & $1.02-1.10$ \\
\hline & & & & -1.71 & .57 & 8.99 & .003 & .18 & \\
\hline
\end{tabular}

Nota: $\chi^{2}=$ Chi cuadrado; $R^{2}=$ Cuadrado de Nagelkerke; $B=$ Coeficiente de regresión; E.T.= Error estándar; Wald $=$ Prueba de Wald; $p=$ Probabilidad; $O R=$ Odd ratio; $I . C .=$ Intervalo de confianza al 95\%; $\mathrm{CVE}=$ Capacidad Visual-Espacial; CIMA= Construcción de Imágenes Mentales por Asociación; UIPE= Uso de Imágenes Predictivas por Experiencia.

\section{DISCUSIÓN}

El primer objetivo del trabajo consistía en analizar si existen diferencias estadísticamente significativas en las PP, DP, EP y el total del PCI y las dimensiones de la SUIS (CVE, CIMA y UIPE), así como el total de la escala, en universitarios españoles. Los hallazgos encontrados muestran que los universitarios españoles con altas puntuaciones en DP, EP y PAP total puntúan significativamente más alto en todos los factores, así como el total de la SUIS.

Del mismo modo, el segundo objetivo consistía en analizar la probabilidad de presentar PP, DP y EP o PAP en general en función de CVE, CIMA y UIPE, así como el total de la SUIS. En este sentido, Ios resultados hallados mostraron que conforme aumenta la puntuación en las distintas dimensiones de la SUIS, aumenta la probabilidad tanto de presentar DP, como EP y PAP en general.

Un reciente estudio, medido desde futuros profesores españoles, halló tres grupos de PAP con baja, moderada y alta intensidad. El grupo de moderada y alta intensidad puntuaron más alto en todos los factores de la SUIS, así como el total de la escala que aquellos futuros profesores con baja intensidad de PAP. Sin embargo, este ha sido el único estudio observado que evalúa la relación entre PAP y SUIS. Los autores del estudio argumentaron que altos niveles de perfeccionismo se asociaban a imágenes mentales intrusivas, lo que creaba una rumia y angustia en estos sujetos (Lee et al., 2011), Io que indica tomar conciencia sobre la importancia de conocer cuál es el UEIP en sujetos con PAP.

No obstante, desde este estudio, se destaca que las PP carecen de significancia con respecto al UEIP. Esto puede ser debido a que las PP, medidas desde el rasgo perfeccionista, son la parte más insana del perfeccionismo 
por su asociación con variables de carácter negativo (Chemisquy, 2017), y esto puede alertarnos de ciertos detaIles a favor del vínculo entre los PAP y el UEIP. Cabe destacar que, el UEIP es un amplificador emocional (Andrade et al., 2013), sin embargo, este amplifica tanto emociones positivas como negativas (Daniels-McGhee y Davis, 1994; Vassilopoulos y Moberly, 2013). Esto quiere decir que la influencia que tiene el UEIP sobre el comportamiento humano, si es regulada adecuadamente también puede ir en beneficio del sujeto, como explican otros estudios (Holmes et al., 2016). De ahí la importancia de seguir estudiando el vínculo entre el UEIP y los PAP, con objeto de diseñar estrategias de medida que prevengan o desciendan la base rumiante y angustia de estos pensamientos perfeccionistas (Lyubomirsky et al., 2015; Vicent et al. 2019).

Pese a ello, es importante tener en cuenta algunas limitaciones del estudio. Por una parte, la muestra no debe ser generalizada teniendo en cuenta que se trata de futuros docentes, por lo que futuros estudios deberían ampliar tanto la edad de la muestra como otros colectivos profesionales. Asimismo, con objeto de comprobar si los datos se replican, el estudio debería ampliarse a otra muestra cultural y población clínica.

\section{CONCLUSIONES}

No obstante, aun presentando estas limitaciones, este estudio es el primero que revisa la capacidad predictiva entre ambas variables y amplía la investigación científica en el campo educativo y artístico. De ahí las implicaciones que puede acarrear en el diseño de tratamientos preventivos y reductores de los PAP y sus comorbilidades (Flett et al., 2012; Lyubomirsky et al., 2015).

\section{REFERENCIAS BIBLIOGRÁFICAS}

Álvarez-Teruel, J.D., Jiménez-Ayala, C.E., Cargua-García, N.I., Aparicio-Flores, M.P., Fernández-Sorgob, A., y García-Fernández, J.M. (2021). Relationship between school anxiety and perfectionism in a sample of Ecuadorian students. Revista Espacios, 42(2), 49-62. https://doi.org/10.48082/espacios-a21v42n07p04

Andrade, J., May, J., Deeprose, C., Baugh, S. J. y Ganis, G. (2013). Assessing vividness of mental imagery: the Plymouth Sensory Imagery Questionnaire. British Journal of Psychology, 105(4), 547-563. https://doi.org/10.1111/bjop.12050

Aparicio-Flores, M.P., Esteve-Faubel, J.M., Esteve-Faubel, R.P., y Granados-Alós, L. (2020). Profiles of perfectionism ruminations in undergraduates: impact on the spontaneous use of mental images. International Journal of Environmental Research and Public Health, 17, 1-8. https://doi.org/10.3390/ijerph17103488

Aparicio-Flores, M.P., Esteve-Faubel, J.M., Vicent, M., y García-Fernández, J.M. (2018). Validación española de la Spontaneous Use of Imagery Scale y perfiles del uso espontáneo de imágenes mentales con empatía disposicional Manuscrito no publicado.

Chemisquy, S. (2017). Revisión teórica sobre el perfil cognitivo del perfeccionismo desadaptativo. Revista de Investigación Apuntes Universitarios, 16-40. https://doi.org/10.17162/au.v8i1.177

Chemisquy, S. (2017). Revisión teórica sobre el perfil cognitivo del perfeccionismo desadaptativo. Revista de Investigación Apuntes Universitarios, 16-40. https://doi.org/10.17162/au.v8i1.177

Daniels-McGhee, S. y Davis, G. A. (1994). The imagery-creativity connection. Journal of Creative Behavior, 28 (3), 151-176. https://doi.org/10.1002/j.2162.6057.1994.tb01189.x

Esteve-Faubel, J.M., Aparicio-Flores, M.P., Vicent, M., Gonzálvez, C., Sanmartín, R., y García-Fernández, J.M. (2020). Validation of Spanish version of the Perfectionism Cognitions Inventory: Profiles of automatic perfectionism thoughts and their associations with social anxiety. Professional Psychology: Research and Practice, 51(3), 268-277. https://doi.org/10.1037/pro0000290

Fearn, M., Marino, C., Spada, M.M., y Kolubinski, D.C. (2021). Self-critical rumination and associated metacognitions as mediators of the relationship between perfectionism and self-esteem. Journal of Rational-Emotive \& Cognitive-Behavior Therapy, 1-20. https://doi.org/10.1007/s10942-021-00404-4

Flett, G. L., Molnar, D. S., Nepon, T. y Hewitt, P. L. (2012). A mediational model of perfectionistic automatic 
thoughts and psychosomatic symptoms: The roles of negative affect and daily hassles. Personality and Individual Differences, 52 (5), 565-570. https://doi.org/10.1016/j.paid.2011.09.010

Garcia-Fernandez, J. M., Ingles, C. J., Suria, R., Lagos-San Martín, N., Gonzálvez, C., Aparisi,D.y MartinezMonteagudo, M. C. (2015). Profiles of emotional intelligence and learning strategies in a sample of Chilean students. European Journal of Psychology of Education, 30, 437-455. D0I: 10.1007/S10212-015-0254-9

Hewitt, P. L. y Genest, M. (1990). The ideal self: schematic processing of perfectionistic content in dysphoric university students. Journal of Personality and Social Psychology, 59 (4), 802-808. https://doi.org/10.1037/0022-3514.59.4.802

Holmes, E.A., Bonsall, M.B., Hales, S.A., Mitchell, H., Renner, F., Blackwell, S.E., Watson, P., Goodwin, G.M., y Di Simplicio, M. (2016). Applications of time-series analysis to mood fluctuations in bipolar disorders to promote treatment innovation: a case series. Translational Psychiatry, 6, 1-10.

Kosslyn, S. M., Chabris, C. F., Shephard, J. M. y Thompson, W. L. (1998). Spontaneous Use of Imagery Scale (SUIS). Manuscrito inédito.

Lee, M., Roberts-Collins, C., Coughtrey, A., Phillips, L., y Shafran, R. (2011). Behavioural expressions, imagery and perfectionism. Behavioural Cognitive Psychotherapy, 39, 413-425.

Lyubomirsky, S., Layous, K., Chancellor, J., y Nelson, K. S. (2015). Thinking about rumination: the scholarly contributions and intellectual legacy of Susan Nolen-Hoeksema. Annual Review of Clinical Psychology, 11, 122. https://doi.org/10.1146/annurev-clinpsy-032814-112733

Ruiz-Esteban, C., Méndez, I., Fernández-Sogorb, A., y Álvarez-Teruel, J.D. (2021). Perfectionism classes and agression in adolescentes. Frontiers in Psychology, 12(686380), 1-7. https://doi.org/10.3389/fpsyg.2021.686380

Mendez, F.X., Espada, J.P., Orgiles, M., Hidalgo, M.D. y Garcia-Fernandez, J. M. (2008). Psychometric properties and diagnostic ability of the separation anxiety scale for children (SASC). European Child \& Adolescent Psychiatry, 17, 365-372. D0I: 10.1007/S00787-008-0678-8

Mohammadi, A., y Roshan-Chasli, R. (2020). The relationship between perfectionism and social anxiety symptoms with self-compassion mediation among students studying at shahed university. Biquarterly Journal of Cognitive Strategies in Learning, 8(15), 161-181. https://doi.org/10.22084/J.PSYCHOGY.2020.20782.2087

Nelis, S., Holmes, E. A., Griffith, J. W. y Raes, F. (2014). Mental imagery during daily life: psychometric evaluation of the Spontaneous Use of Imagery Scale (SUIS). Psychologica Belgica, 54 (1), 19-32. https://doi.org/10.5334/pb.ag

Vassilopoulos, S. P., y Moberly, N. J. (2013). Cognitive bias modification in preadolescent children: inducing and interpretation bias affects self-imagery. Cognitive Therapy and Research, 37 (3), 547-556. https://doi.org/10.1007/s10608-012-9481-4

Vicent, M., Rubio-Aparicio, M., Sanchez-Meca, J. y Gonzalvez, C. (2019). A reliability generalization meta-analysis of the child and adolescent perfectionism scale. Journal of Affective Disorders, 245, 533-544. https://doi.org/10.1016/j.jad.2018.11.049

Vicent, M., Sanmartín, R., Gonzálvez, C., Vásconez-Rubio, 0., y García-Fernández, J.M. (2021). Perfectionism, motives and barriers to exercise from a person-oriented-approach. International Journal of Environmental Resourch Public Health, 18(15), 8125. https://doi.org/10.3390/ijerph18158125

Wigert, B., Reiter-Palmon, R., Kaufman, J. C., y Silvia, P. J. (2012). Perfectionism: the good, the bad, and the creative. Journal of Research in Personality, 46 (6), 775-559. https://doi.org/10.1016/j.jp.2012.08.007 